Athens, and the Department of Neurology, Medical College of Georgia, Augusta, and the Athens Magnetic Imaging. The genu of the corpus callosum was significantly smaller in the dyslexic children. Familial left-handedness, and ADD with and without hyperactivity distinguished the dyslexic children from controls. (Hynd GW et al. Dyslexia and corpus callosum morphology. Arch Neurol January 1995;52:32-38). (Respond: Dr George W Hynd, Center for Clinical and Developmental Neuropsychology, 570 Aderhold Hall, The University of Georgia, Athens, GA 30602).

COMMENT. Studies of MRI morphology of the corpus callosum in monozygotic twins at Dartmouth Medical School Program in Cognitive Neuroscience showed wide variations in size and shape of the human corpus callosum. Measurements revealed greater similarity in twin pairs than in randomly paired controls. (Ann Neurol 1989;26:100). The anatomy of the corpus callosum appears to be under genetic control as well as being influenced by nongenetic factors. How much this natural variation in size of the corpus callosum influenced the results of the above study in dyslexia is debatable. (see Progress in Pediatric Neurology I, PNB Publ, 1991, pp 168-9).

\title{
SILVER-RUSSELL SYNDROME AND COGNITIVE DISORDERS
}

Cognitive abilities of 20 boys and 5 girls, aged 6 to 11 years, with SilverRussell syndrome were investigated at the Prince of Wales Hospital, Shatin, Hong Kong, the Institute of Child Health, and Middlesex Hospital, London, UK. The mean full scale IQ was 86 , and $32 \%$ scored $<70$. Reading comprehension was 24 months below chronological age in $40 \%$. Speech therapy was required in $48 \%$. IQ scores were positively correlated with growth in head circumference. (Lai KYC et al. Cognitive abilities associated with the Silver-Russell syndrome. Arch Dis Child Dec 1994;71:490-496). (Respond: Professor D Skuse, Institute of Child Health, London, UK).

COMMENT. Features of Silver-Russell syndrome include low birth weight, short stature, body asymmetry, clinodactyly, and craniofacial dysmorphism - small triangular face, large forehead, small chin, shark's mouth, and low set ears. The present study adds cognitive disorders to the list of features. Intrauterine growth retardation of Silver-Russell syndrome beginning early in pregnancy results in reduction in both birth weight and length. In this "symmetrical" type of growth retardation, in utero brain development is more likely to be affected than when growth retardation begins late in pregnancy.

\section{INFECTIOUS DISORDERS}

\section{CONGENITAL TOXOPLASMOSIS: TREATMENT AND OUTCOME}

Neurologic, cognitive, and motor outcomes for 36 children with congenital toxoplasmosis treated with pyrimethamine and sulfadiazine for 1 year are reported from Michael Reese Hospital, Chicago, IL, and other Centers. Active infection, seizures, and motor abnormalities resolved in most during therapy. Of 29 infants evaluated at 1 year of age, 23 (79\%) had a Mental Developmental Index of 102, and 6 had scores $<50$. Sibling controls had higher scores than patients, but sequential IQ testing showed no deterioration over time. Six of eight children with obstructive hydrocephalus relieved by shunts 